\title{
New Definition and Classifications of Epilepsy Among Highlights of the American Epilepsy Society Annual Meeting
}

\author{
Michael R Sperling
}

Chair, 2016 American Epilepsy Society Annual Meeting Committee, Member, AES Council on Education, Baldwin Keyes Professor of Neurology, Vice Chair for Research, Department of Neurology, Sidney Kimmel Medical College of Thomas Jefferson University, Philadelphia, US; Director, Jefferson Comprehensive Epilepsy Center, Philadelphia, us

DOI: https://doi.org/10.17925/USN.2017.13.01.13

$\mathrm{M}$ ore than 5 million children and adults suffer from epilepsy, but diagnosis and treatment remains challenging. A new classification system rolled out at the recent American Epilepsy Society annual meeting should help make diagnosis easier, ensuring earlier identification of epilepsy and more timely treatment. Other cutting-edge research, insights and best practices presented at the meeting focused on surgical and minimally invasive treatment options, the use of anti-epileptic drugs in pregnant women, the potential benefits of cannabidiol oil in patients refractory to standard treatment and the underdiagnosis of infantile spasms.

\section{Keywords}

Nervous system diseases, seizure disorder, myoclonic epilepsy, infantile spasms, cannabidiol, cannabis plant, epileptic seizure, patient care, epilepsy research, epilepsy treatment, recurrent seizures, unprovoked seizure, temporal lobe, uncontrolled seizures, marijuana and epilepsy.

\section{Disclosure: Michael R Sperling discloses receiving support as Contract Research from NIH, DARPA, UCB Pharma, Eisai, Sunovion, SK Life Sciences, Glaxo, Upsher-Smith, Acorda, Medtronics, Marinus, Brain Sentinel Pfizer (all payments to Thomas Jefferson University); Other Services member of Board of Directors of the Epilepsy Foundation of Eastern PA, Editor in Chief of Epilepsia, member of the ILAE Executive committee. This article is a report of congress highlights and has not been submitted to external peer reviewers. No funding was received for the publication of this article.}

Authorship: All named authors meet the International Committee of Medical Journal Editors (ICMJE) criteria for authorship of this manuscript, take responsibility for the integrity of the work as a whole, and have given final approval to the version to be published.

Open Access: This article is published under the Creative commons Attribution Noncommercial License, which permits any noncommercial use, distribution, adaptation, and reproduction provided the original author(s) and source are given appropriate credit.

Received: January 16, 2017

Published Online: March 1, 2017

Citation: US Neurology, 2017;13(1):13-5

Corresponding Author: Michael R Sperling Thomas Jefferson University, 901 Walnut St, Dept of Neurology Ste 400, Philadelphia, PA 19107, US.

E: Michael.Sperling@jefferson.edu
As its mission states, the American Epilepsy Society (AES) is working to eradicate epilepsy and its consequences. While this lofty goal may not yet be visible on the horizon, researchers and physicians who treat patients with epilepsy are making progress, as evidenced by the research and latest practices presented at the AES 70th Annual Meeting, held December 2-6, 2016 in Houston, US. It is the largest professional gathering for epilepsy in the world. Notable was the announcement of new classifications of epilepsy, the diagnosis of which confounds many in the nonmedical and medical world. Following on the heels of the revised 2014 definition of epilepsy, the hope is that this clarification and simplification of terminology will help physicians identify patients with epilepsy earlier, resulting in appropriate treatment sooner to prevent some of the more devastating consequences of the condition. Other highlights of the meeting ranged from the latest in epilepsy surgery to discussions of the challenges of treating women with epilepsy who are of childbearing age in light of the risks of anti-epileptic drugs (AEDs) to the fetus.

\section{Epilepsy-new definition and classifications}

The AES Annual Fundamentals Symposium was dedicated to discussion of the new definition and classification of epilepsy, featuring leading researchers. It was chaired by Robert Fisher, MD, PhD, of the Stanford University School of Medicine. Dr. Fisher led the project to develop a formal definition and update the seizure types." Jacqueline French, MD, of New York University School of Medicine provided insight into the new definition of epilepsy. ${ }^{2}$ She noted that while epilepsy was until recently defined as the occurrence of two or more epileptic seizures, the 2014 International League Against Epilepsy (ILAE) revision states that a diagnosis of epilepsy now can be made after one epileptic seizure if there is a probability of further seizures. Ideally, this will move patients who have an initial seizure towards more timely diagnosis and effective treatment.

Dr. Fisher provided a detailed explanation of the ILAE classification of seizures, which may help improve patient care. ${ }^{1}$ He noted the goal is to make determining type easier for seizures that do not fit into any prior categories, clarify what is meant when a seizure is said to be of a particular type and provide greater transparency of terminology to the non-medical community. For example, instead of being described as "complex partial seizures," those that arise in one area of the brain will now be called "focal seizures with impaired awareness." The effect of the seizure is quite clear with the new nomenclature, which may help physicians, patients and family members better understand the seizures. In addition to updating terminology, other changes are: to allow for the possibility that some seizure types (such as tonic seizures) may be either focal or generalized in onset, allow for seizures whose onset is unknown, clarify "impairment of consciousness," include previously unclassified types of seizures, validate use of 
supportive information (for example electroencephalogram [EEG]), conform with International Classification of Diseases (ICD) 11 and 12 terminology, update the 2001 glossary of seizure terms, standardize common descriptors of seizures, and map old to new terms.

He provided numerous examples of the new classification system at work. For example, a 25-year-old woman said her seizures began with 30 seconds of an intense feeling that "familiar music is playing." While she could hear people talking, she later realized she could not determine what they were saying. Afterward, she was mildly confused and had to reorient herself. Previously, this patient would have been diagnosed with complex partial seizures, while under the new system she would be diagnosed with focal seizures of impaired awareness. In another example, a man's wife witnessed her husband having a seizure in bed, including bilateral stiffening followed by bilateral shaking. An EEG showed a clear right parietal slow wave focus, and an MRI showed a right parietal region of cortical dysplasia. The old classification would have been partial onset, secondarily generalized seizures. The new classification would be focal to bilateral tonic-clonic seizures.

The full ILAE classification guidelines will be published in Epilepsia this year.

\section{Surgery and minimally invasive options for epilepsy} While AEDs typically are first-line therapy for epilepsy, there are a variety of procedural options as well, from surgery to a host of minimally invasive procedures, including thermal ablation (radiofrequency, laser interstitial thermal ablation and focused ultrasound), radiosurgery, electrical neuromodulation, drug infusion and gene therapy. The Epilepsy Specialist symposium focused on determining the best course for individual patients.

Temporal lobe epilepsy is the most common form of localized epilepsy. compared to surgery, the minimally invasive options for this form of epilepsy result in less morbidity and neurocognitive deficit, but they also are less effective. During this session, Robert E Gross, MD, PhD, of Emory University School of Medicine ${ }^{3}$ and Michael Sperling, MD, of Thomas Jefferson University, discussed that, while anterior temporal lobectomy is the most common surgery for this type of epilepsy, it could be supplanted in part by thermal ablation using a laser device. ${ }^{4}$ More data are needed but a new trial has begun that hopefully will better define the benefits and risks of this procedure.

Hypothalamic hamartoma is a rare pediatric brain tumor that triggers gelastic seizures that cause laughing. Treatment is challenging, ranging from surgery - which is invasive and risky - to radiation, which has significant side effects. Daniel J Curry, MD, of Baylor College of Medicine discussed using thermal ablation to treat this condition. ${ }^{5}$ It holds great promise in revolutionizing treatment for these children. He noted it is safer than open surgery and appears to be effective.

The Hot Topic Symposium featured two talks on the Radiosurgery or Open Surgery for Epilepsy (ROSE) trial of 234 patients randomized to receive either gamma knife therapy or anterior temporal lobectomy for the treatment of mesial temporal lobe epilepsy, given by Nicholas M Barbaro, MD, of the University of California San Francisco, ${ }^{6}$ Mark Quigg, MD, of the University of Virginia, Edward F Chang, MD, University of California at San Francisco ${ }^{6}$ and Donna K Broshek, PhD, of the University of Virginia.? Despite hopes that this less invasive treatment would be an equally effective and safer option than surgery for patients with mesial temporal lobe epilepsy, the trial found that the gamma knife offered no advantages over open surgery. In fact, there appeared to be disadvantages regarding efficacy. After 25 to 36 months, 52\% of patients who received radiosurgery were seizure-free, versus. $78 \%$ of open surgery patients. Additionally, adverse effects such as memory and language loss were no better with radiosurgery than open surgery. The procedure might be suitable for patients who refuse surgery as long as they fully understand options, including thermal ablation and open surgery, and their various advantages and disadvantages.

\section{Risks of pregnancy and anti-epileptic drugs}

Women with epilepsy often want to have children. However, AEDs confer risks to the fetus and may lead to birth defects. Experts speaking at the North American Commission Symposium tackled the controversies in the management of epilepsy during pregnancy. Torbjörn Tomson, MD, PhD, of the Karolinska Institute, Stockholm, Sweden, noted that AEDs increase the risk of fetal defects. ${ }^{8}$ The risks are highest for monotherapy with valproate, which is associated with major congenital malformations, such as cardiac anomalies, neural tube defects, cleft palate and cleft lip and hypospadia. Studies have found the risk of having a baby with a major congenital malformation reaches nearly $10 \%$ in women on valproate. Higher doses are associated with greater risk. ${ }^{9}$ One study found that women on AEDs whose previous pregnancy resulted in major fetal malformation had a $35.7 \%$ chance of a major malformation in the subsequent pregnancy if they remained on the same AED, whereas that risk decreased to $3 \%$ if they were not taking that same AED. ${ }^{10}$ Valproate can also affect cognitive development and increase the risk of autism and autism spectrum disorder.

Due to these risks, the use of valproate has decreased significantly over time. However, physicians and their patients also must consider the risk of epileptic seizures for the patient and her fetus. The risk of uncontrolled seizures is significant, and in some cases there are few effective alternatives for some women with idiopathic or genetic generalized epilepsy.

It is vital that women with epilepsy carefully plan their treatment course prior to becoming pregnant. Kimford J Meador, MD, of Stanford University, noted that half of pregnancies are not planned, so epilepsy specialists must discuss the risks of AEDs with patients of childbearing age. ${ }^{11}$ Women should receive informed consent outlining the significant known and unknown risks prior to conceiving. For example, some AEDs decrease hormone levels. Dr. Meador emphasized that valproate often is a suboptimal first choice of AED for women with epilepsy who are of childbearing age and other agents are usually preferred. As previously noted, however, valproate may be the only AED that fully controls their seizures for some people. In cases where valproate is necessary, the lowest dose possible should be prescribed. As is evident, more research is needed regarding the risks of AEDS, particularly regarding the cognitive effects of fetal and neonatal exposure, as well as the effects of combinations of medications.

\section{Potential benefits of cannabidiol}

Several abstracts reported on the growing evidence that an oral formulation of pharmaceutical-grade cannabidiol (CBD) may be beneficial for some patients with epilepsy. Randomized, double-blind, placebo-controlled trials found $20 \mathrm{mg} / \mathrm{kg}$ per day of CBD reduced the frequency of seizures in children and adults with certain treatment-resistant forms of epilepsy. A purified molecule from the cannabis plant, CBD has less than $1 \%$ tetrahydrocannabinol (THC), so it does not produce psychoactive effects. Among the research were studies presented by Elizabeth A Thiele, MD, 
PhD, of Harvard Medical School, which found significant benefit in reduced seizure severity, including a 39\% reduction in patients with Dravet syndrome and $44 \%$ in those with Lennox-Gastaut syndrome. ${ }^{12,13} \mathrm{~A}$ small open-label study presented by Jerzy P Szaflarski, MD, PhD, of the University of Alabama found CBD provided a 50 percent reduction in seizure severity in a majority of patients with various forms of treatment-resistant epilepsy. ${ }^{14}$ However, not all patients benefitted from CBD and some worsened; moreover, side effects, particularly sedation, are common. Dr. Theile noted that CBD is not a silver bullet, but it may be helpful for some children who do not benefit from standard drugs. ${ }^{12,13}$ The product is expected to be submitted to the Food and Drug Administration (FDA) for approval as a prescription medication this year or next.

\section{Infantile spasms often not diagnosed promptly}

A small University of California Los Angeles study found nearly half of babies who suffer from infantile spasms are not accurately diagnosed for more than a month after the seizures start. ${ }^{15}$ Researchers surveyed the parents of 100 children who suffered from infantile spasms and found only $29 \%$ were seen by a healthcare provider who accurately diagnosed them within one week of spasm onset. Several infants remained undiagnosed for years. That delay may lead to serious consequences, including intellectual disability, autism and death. About one-third of children in the study suffered a 15-point reduction in IQ due to the delay, researchers estimate. Many parents said they knew something was wrong, but their concerns often were discounted by pediatricians, emergency department physicians and even neurologists. In many cases, they eventually diagnosed their children themselves using internet sources. Successful treatment depends on prompt diagnosis. Children with signs of infantile spasms - which can be subtle and look like a cluster of small jerks or a quick drop of the head - should be seen by a pediatric neurologist and assessed via an overnight or 24-hour video EEG.

\section{Conclusion}

We have made significant gains in the diagnosis and treatment of epilepsy, but much more work remains to be done. We look forward to the 2017 AES Annual Meeting in Washington DC, December 1-5, and to learning more about continued steady progress in treatment and diagnosis, such as improvements in the identification and validation of biomarkers in epilepsy. $\square$
1. Fisher R, New Classification of Seizure Types, Presented at: American Epilepsy Society Annual Meeting, Houston, US, December 2,2016.

2. French J, New Definition of Epilepsy, Presented at: American Epilepsy Society Annual Meeting, Houston, US, December 2, 2016

3. Gross R, Mesial Temporal Lobe Epilepsy: Minimal. Presented at: American Epilepsy Society Annual Meeting, Houston, US, December 2, 2016.

4. Sperling M, Mesial Temporal Lobe Epilepsy: Maximal. Presented at: American Epilepsy Society Annual Meeting, Houston, US, December 2, 2016.

5. Curry D, Hypothalamic Hamartomas: One Disorder Treated by Multiple Approaches, Presented at: American Epilepsy Society Annual Meeting, Houston, US, December 2, 2016.

6. Barbaro N, Quigg M, Chang E. Radiosurgery or Open Surgery for Epilepsy (ROSE). Presented at: American Epilepsy Society Annua
Meeting, Houston, US, December 6, 2016.

Broshek D, Neuropsychological Outcome. Presented at American Epilepsy Society Annual Meeting, Houston , US, December 6, 2016.

8. Tomson T, The Valproate Controversy: The Worldwide Perspective Presented at: American Epilepsy Society Annual Meeting, Houston, US, December 3, 2016.

9. Tomson T, Major congenital malformations in children of women with epilepsy. Seizure. 2015 May;28:46-50.

10. Vajda FJ, Teratogenesis in repeated pregnancies in antiepileptic drug-treated women. Epilepsia. 2013 Jan;54(1):181-6.

11. Meador KJ, Pre-pregnancy Planning: AED Choice and Pregnancy Outcomes, Presented at: American Epilepsy Society Annual Meeting, Houston, US, December 3, 2016.

12. Cross JH, Cannabidiol (CBD) Reduces Convulsive Seizure Frequency in Dravet Syndrome: Results of a Multi-Centered, Randomized,
Controlled Study (GWPCARE1), Presented at American Epilepsy Society Annual Meeting, Houston, US, December 4, 2016.

13. Thiele $E$, Cannabidiol (CBD) Significantly Reduces Drop Seizure Frequency in Lennox-Gastaut Syndrome: Results of a MultiCenter, Randomized, Double-Blind, Placebo-Controlled Trial (GWPCARE4), Presented at American Epilepsy Society Annual Meeting, Houston, US, December 3, 2016

14. Szaflarski J, Response to Pharmaceutical Grade Cannabidiol in a State-Sponsored Treatment Program, Presented at American Epilepsy Society Annual Meeting, Houston, US, December 3, 2016.

15. J. Lay, E Cheng J. Weng Y Eliyan, E Schmid, P. Hung J. Helali, A. Weng, R. Elsanadi, A. Chien, T. Cao, O. Hayward, R. Sankar, C. Baca, S. Hussain. Significant Delay in Diagnosis and Treatment of Infantile Spasms is Common. Presented at: American Epilepsy Society Annual Meeting, Houston, US, December 4, 2016. 